\title{
Multifunctional Separation Mechanism on Poly(oxyethylene) Stationary Phases in Capillary Liquid Chromatography
}

\author{
Toyohide TAKEUCHi ${ }^{\dagger}$ and Lee Wah LIM \\ Department of Chemistry, Faculty of Engineering, Gifu University, 1-1 Yanagido, Gifu 501-1193, Japan
}

\begin{abstract}
The retention behavior on poly(oxyethylene) (POE) chemically-bonded stationary phases was investigated in ion-exchange, reversed-phase (RP) and hydrophilic interaction (HILIC) modes in capillary liquid chromatography. The eluent cations fixed on the oxygen atoms of the POE chains by ion-dipole interaction worked as the anion-exchange sites for the separation of inorganic anions. Nonpolar analytes were retained on the POE stationary phases in the RP mode, while polar organic analytes were retained in the HILIC mode. Sodium alkyl sulfate dynamically modified on the stationary phase enhanced the hydrophobic property of the stationary phase. The effect of the oxyethylene unit number on the retention behavior was examined, and it was expected that the eluent cation is trapped among multiple POE chains by ion-dipole interaction.
\end{abstract}

(Received May 10, 2010; Accepted July 8, 2010; Published September 10, 2010)

\section{Introduction}

Various stationary phases have been developed in ion chromatography since its introduction in $1975 .^{1}$ Most stationary phases employed in ion chromatography have functional groups with charged or chargeable moieties, where ionic analytes experience electrostatic attractive or repulsive forces.

Inorganic anions could also be separated even if the stationary phase possesses no ion exchange site. For example, poly(ethylene glycol) (PEG) with an average molecular mass of $10000-20000$ dynamically coated on a hydrophobic triacontyl-functionalized silica (C30) resolved inorganic anions in the partition mode, where the retention of iodide increased with increasing eluent concentration. ${ }^{2}$ Inorganic anions could also be separated in the partition mode on a C30 modified with poly(oxyethylene) oleyl ether (POEOE) with an average degree of polymerization of 50 (nominal average molecular mass of 2468). ${ }^{3}$ Both cation and anion of the eluent affected the retention of analyte anions on the above PEG and POEOE stationary phases. This is because analyte anions partitioned into the above stationary phase with the eluent cation, and hydration strength of the eluent cation and anion could affect the hydrophobicity of the stationary phase. It is speculated that more hydrating eluent ions can withdraw more water from the PEG or POEOE stationary phase into the eluent zone, leading to more hydrophobic properties of the stationary phase.

Contrarily, as for a dodecaoxyethylene (12OE)-bonded silica column, ion exchange was involved for the retention of analyte anions, considering the retention behaviors of analyte anions as well as the results of indirect detection of analyte anions. ${ }^{4}$ It was expected that the eluent cations fixed on the oxygen atoms of the $12 \mathrm{OE}$ moieties by ion-dipole interaction worked as the anion-exchange sites.

$\dagger$ To whom correspondence should be addressed.

E-mail: take-t@gifu-u.ac.jp
Guo et al. ${ }^{5}$ introduced a PEG stationary phase for reversedphase (RP) liquid chromatography (LC) and separated phenyl compounds and traditional Chinese medicine. PEG-bonded silica-based columns, e.g., Discovery HS PEG column (Supelco; Bellefonte, PA), are now commercially available, and they have been applied to the analysis of natural products. ${ }^{6,7}$ In addition, it is known that the PEG moiety could form a coil- or helix-like conformation in the organic-aqueous media, ${ }^{8}$ leading to generation of different selectivity values compared with common C18 stationary phases.

Trapping of eluent cations on the 12OE-bonded stationary phase is similar to trapping of potassium ion into 18-crown 6-ether, but the former is more flexible, and the size of the cation may not be very significant. ${ }^{4}$ Lamb and Smith prepared stationary phases bonded with crown ether or cryptand for the separation of alkali and other metal cations, where the analyte cations are trapped by ion-dipole interactions. ${ }^{9}$ Furthermore, they found that anions were also separated through the formation of ion pairs with the bound cations. The present paper investigates the retention behavior on POE-bonded stationary phases in ion-exchange, RP and hydrophilic interaction (HILIC) modes in capillary LC.

\section{Experimental}

\section{Reagents and materials}

Reagents employed were of guaranteed reagent grade and were obtained from Wako Pure Chemical Industries (Osaka, Japan), unless otherwise noted. $\mathrm{N}$-Hydroxysuccinimido-36-methoxy-4,7,10,13,16,19,22,25,28,31,34-undecaoxaoctricontanoate (Methyl-12OE-NHS ester) and $\mathrm{N}$-hydroxysuccinimido12-methoxy-4,7,10-trioxadodecanoate (Methyl-4OE-NHS ester) were obtained from PIERCE (Rockford, IL). SUNBRIGHT ME-020AS (Methyl-POE-2000-NHS ester) and SUNBRIGHT ME-050AS (Methyl-POE-5000-NHS ester) were obtained from NOF Corporation (Tokyo, Japan), and the average molecular 


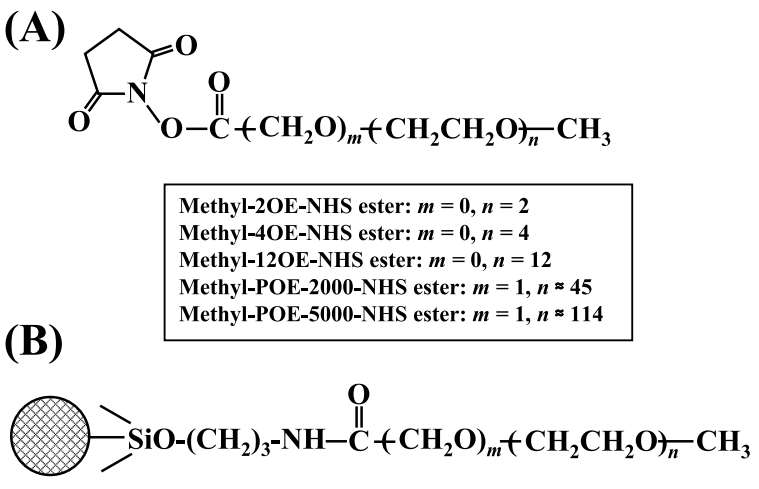

Fig. 1 Structures of the POE-NHS ester reagents employed in this work (A) and the expected chemical structures of the stationary phases (B).

masses given by the manufacturer were 2280 and 5283 , respectively. $\mathrm{N}$-Hydroxysuccinimido-6-methoxy-4-oxahexanoate (Methyl-2OE-NHS ester) was obtained from Quanta Biodesign (Powell, OH). The structures of the POE reagents employed are shown in Fig. 1A, and the expected chemical structures of the stationary phases prepared are shown in Fig. 1B. The numbers of the oxyethylene unit for Methyl-POE-2000-NHS ester and Methyl-POE-5000-NHS ester are calculated from the average molecular weight provided by the manufacturer. Purified water was produced in the laboratory by using a GS-590 water distillation system (Advantec, Tokyo, Japan). HPLC-grade distilled water was used for the RP mode. All solutions used in this work were prepared using the purified water or the HPLC-grade distilled water. Porous 3-aminopropylsilica, TSKgel $\mathrm{NH}_{2}-60(5 \mu \mathrm{m}$ particle diameter, $60 \AA$ mean pore diameter) was taken from the packed column obtained from TOSOH (Tokyo, Japan).

\section{Apparatus}

The chromatographic measurements were carried out by using a capillary LC system constructed by an L.TEX-8301 Micro Feeder (L.TEX Corporation, Tokyo, Japan) equipped with an MS-GAN 050 gas-tight syringe $(0.5 \mathrm{ml}$; Ito, Fuji, Japan) as a pump, a Model M435 microinjection valve with an injection volume of $0.15 \mu \mathrm{l}$ (Upchurch Scientific, Oak Harbor, WA) as an injector, a $0.53-\mathrm{mm}$ i.d. $\times(50-150) \mathrm{mm}$ microcolumn, and a UV-970 UV detector (JASCO, Tokyo, Japan). The UV detector was operated at 210 or $254 \mathrm{~nm}$. A capillary flow cell $(75 \mu \mathrm{m}$; JASCO) was equipped with a UV detector. The data were acquired by a Chromatopac C-R4A data processor (Shimadzu, Kyoto, Japan). The inlet pressure was monitored by an L.TEX8150 Pressure Sensor (L.TEX).

\section{Preparation of POE-bonded stationary phase}

POE-bonded stationary phases were prepared according to the previous work. ${ }^{4}$ A $0.2-\mathrm{g}$ amount of TSKgel $\mathrm{NH}_{2}-60$ was placed in a $20-\mathrm{mL}$ vial. A $0.1-\mathrm{g}$ amount of $N$-hydroxysuccinimide ester reagents of POE were dissolved in $2-3 \mathrm{~mL}$ volume of $0.1 \mathrm{M}$ phosphate buffer ( $\mathrm{pH} 6.7-7.0)$, and the solution was then poured into the vial. The reaction was carried out at room temperature $\left(20-26^{\circ} \mathrm{C}\right)$ for $30 \mathrm{~min}$, followed by washing with deionized water and methanol.

The separation column was prepared from a fused silica capillary tube $(0.53 \mathrm{~mm}$ i.d. $)$ using a slurry packing method previously reported. ${ }^{10}$

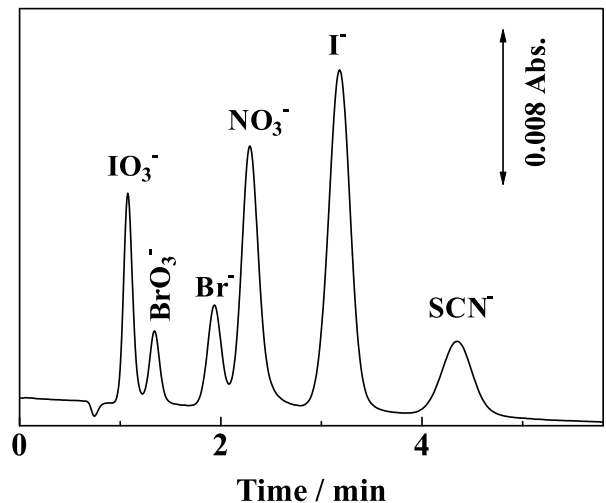

Fig. 2 Separation of inorganic anions on a Methyl-12OE-bonded stationary phase. Column, Methyl-12OE $(50 \times 0.53 \mathrm{~mm})$; mobile phase, $20 \mathrm{mM}$ sodium sulfate; flow rate, $10.0 \mu \mathrm{l} / \mathrm{min}$; wavelength of UV detection, $210 \mathrm{~nm}$; analytes, $0.1 \mathrm{mM}$ each of iodate, bromate, bromide, nitrate, iodide and thiocyanate; injection volume, $0.15 \mu \mathrm{l}$.

\section{Preparation of saliva sample}

A 1.21-g amount of saliva was diluted in $10 \mathrm{~mL}$ of deionized water by using a volumetric flask, and centrifuged at $3000 \mathrm{rpm}$ for $5 \mathrm{~min}$, followed by filtration with a $0.45-\mu \mathrm{m}$ membrane filter. The saliva sample was then stored in a refrigerator.

\section{Results and Discussion}

\section{Ion-exchange mode}

Figure 2 demonstrates the separation of six inorganic anions on a 5-cm Methyl-12OE-bonded column, where UV-absorbing anions are separated in the ion-exchange mode in $5 \mathrm{~min}$. It was indicated in the previous work that the eluent cation is trapped on the oxygen atoms of the Methyl-12OE chain and that the trapped cations worked as the anion-exchange sites. ${ }^{4}$ It was also demonstrated in the previous work that the eluent cation could affect the retention of anions. ${ }^{4}$

The present system was applied to the determination of UV-absorbing anions contained in a saliva sample. Figure 3 demonstrates the separation of inorganic UV-absorbing anions contained in a saliva sample using a $10-\mathrm{cm}$ column, where nitrite and bromide could not be separated under the present operating condition. Nitrate, iodide and thiocyanate could be separated, and the concentration of the anions in the saliva was determined to be $47,2.4$ and $20 \mu \mathrm{g} / \mathrm{g}$ for nitrate, iodide and thiocyanate, respectively.

\section{$R P$ and HILIC modes}

Figure 4 illustrates the retention time of uracil and toluene as a function of acetonitrile concentration in the mobile phase on a Methyl-12OE-bonded phase $(150 \times 0.53 \mathrm{~mm})$. It can be seen that the retention time of uracil increases with increasing acetonitrile concentration in the mobile phase, whereas the retention time of toluene decreases with increasing acetonitrile concentration in the mobile phase and reaches the minimum at the acetonitrile concentration at around $70 \%$. The results show that uracil elutes before toluene at lower acetonitrile concentration (RP mode), whereas it elutes after toluene at higher acetonitrile concentration (HILIC mode).

Figure 5 demonstrates the separation of uracil and toluene with two different mobile phases on the Methyl-12OE-bonded phase $(150 \times 0.53 \mathrm{~mm})$. It can be seen that uracil elutes before 


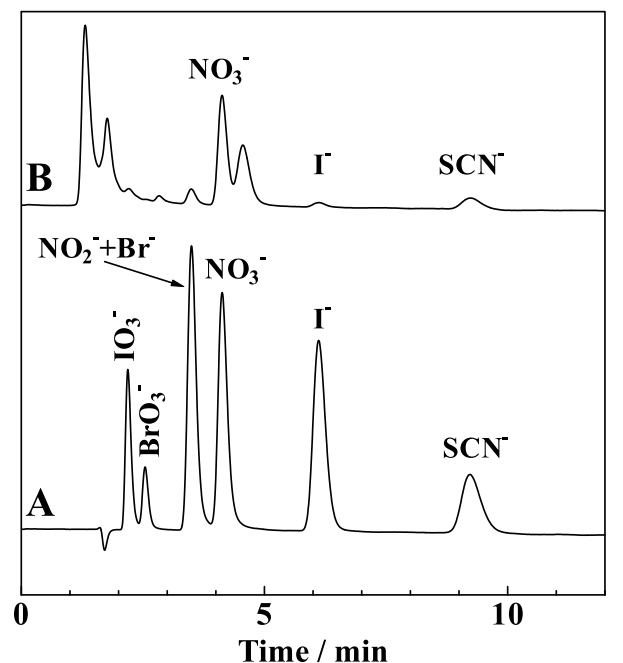

Fig. 3 Separation of inorganic anions in saliva on a Methyl-12OE-bonded stationary phase. Column, Methyl-12OE $(100 \times 0.53 \mathrm{~mm})$; mobile phase, $50 \mathrm{mM}$ sodium sulfate; flow rate, $8.0 \mu \mathrm{l} / \mathrm{min}$; wavelength of UV detection, $210 \mathrm{~nm}$; samples, $0.2 \mathrm{mM}$ each of iodate, bromate, nitrite, bromide, nitrate, iodide and thiocyanate (A), saliva sample (B); injection volume, $0.15 \mu \mathrm{l}$.

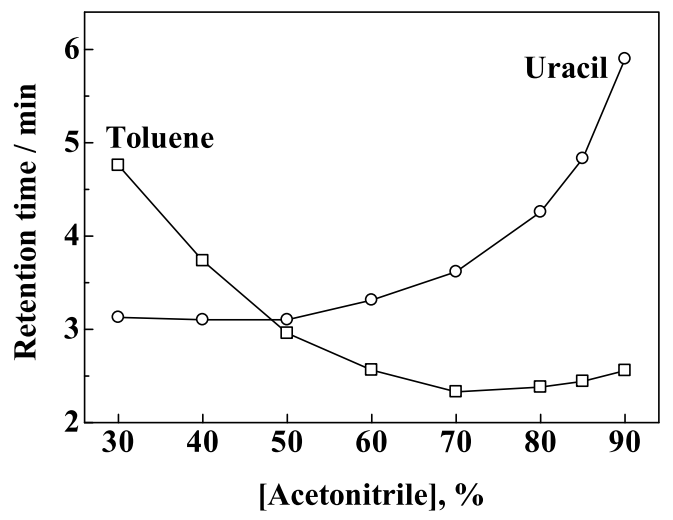

Fig. 4 Retention times of uracil and toluene as a function of acetonitrile concentration in the mobile phase. Column, Methyl-12OE $(150 \times 0.53 \mathrm{~mm})$; mobile phases, acetonitrile-water; flow rate, 8.0 $\mu 1 / \mathrm{min}$; wavelength of UV detection, $210 \mathrm{~nm}$; analytes, $50 \mathrm{mg} / \mathrm{l}$ each of uracil and toluene; injection volume, $0.15 \mu \mathrm{l}$.

toluene in the RP mode, whereas uracil elutes after toluene in the HILIC mode. In addition, narrower peaks are observed for the HILIC mode, compared with those for the RP mode. One of the reasons for the poor efficiency observed for the RP mode may be that the acetonitrile composition is too low (30\%) for toluene.

The separation of uracil and three aromatic hydrocarbons on the Methyl-12OE column in the RP mode is demonstrated in Fig. 6. Uracil, naphthalene, anthracene and pyrene are eluted in this order within $8 \mathrm{~min}$ using a $40 \%$ acetonitrile aqueous solution as the mobile phase.

The hydrophobicity of the POE-bonded stationary phases can be enhanced by passing the eluent containing sodium alkylsulfate. This is because the sodium ion is trapped by the POE chain and alkylsulfate is then trapped as the counter ion. The modification with alkylsulfate is accomplished either by

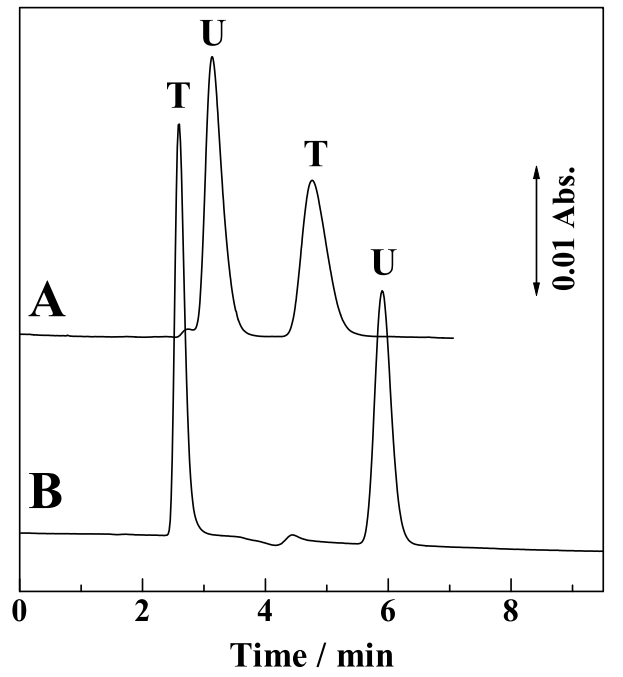

Fig. 5 Separation of uracil and toluene in the RP mode (A) and the HILIC mode (B). Mobile phases: acetonitrile-water (30:70) for A and acetonitrile-water (90:10) for B. Analytes: uracil (U) and toluene (T); other operating conditions as in Fig. 4.

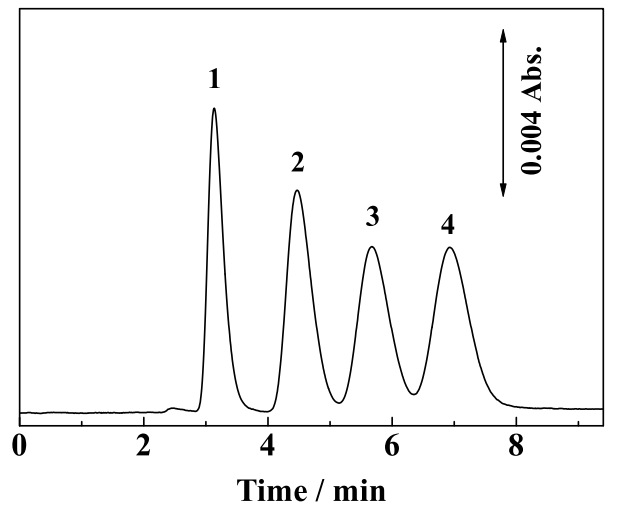

Fig. 6 Separation of uracil and aromatic hydrocarbons in the RP mode on unmodified Methyl-12OE stationary phase. Column, Methyl-12OE $(150 \times 0.53 \mathrm{~mm})$; mobile phase, acetonitrile-water (40:60); flow rate, $8.0 \mu \mathrm{l} / \mathrm{min}$; wavelength of UV detection, $254 \mathrm{~nm}$. Analytes: $10 \mathrm{ppm}$ uracil (1), $31 \mathrm{ppm}$ naphthalene (2), $2.6 \mathrm{ppm}$ anthracene (3), $7.8 \mathrm{ppm}$ pyrene (4); injection volume, $0.15 \mu 1$.

passing sodium alkylsulfate solution into the POE column prior to the separation or by incorporating sodium alkylsulfate in the mobile phase. The latter approach needs a longer time to modify the POE stationary phase. Figure 7 demonstrates the separation of uracil and aromatic hydrocarbons using a 50\% acetonitrile aqueous solution as the mobile phase in the RP mode on unmodified (A) and modified Methyl-12OE stationary phases (B and $\mathrm{C}$ ) using a mixture of acetonitrile and water (50:50) as the mobile phase. Figure $7 \mathrm{~B}$ was obtained on the Methyl-12OE stationary phase modified with $0.5 \mathrm{ml}$ aqueous solution of $20 \mathrm{mM}$ sodium dodecylsulfate prior to the separation, whereas Fig. 7C was obtained on the Methyl-12OE stationary phase modified with $0.5 \mathrm{ml}$ methanol solution of $20 \mathrm{mM}$ sodium octadecylsulfate. It can be seen that the retention of aromatic hydrocarbons was increased by modification with alkylsulfate. In addition, although it was expected that the retention time of the aromatic hydrocarbons on the Methyl-12OE stationary 


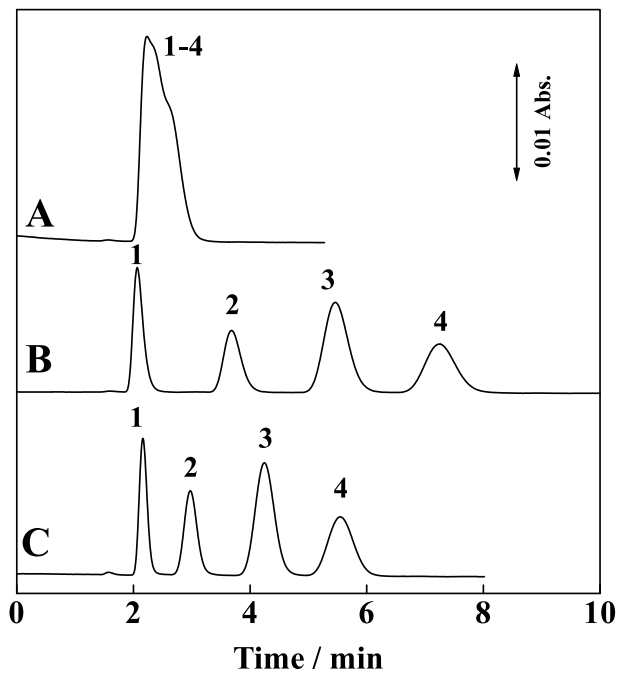

Fig. 7 Separation of uracil and aromatic hydrocarbons in the RP mode on unmodified and modified Methyl-12OE stationary phases. Column, Methyl-12OE $(100 \times 0.53 \mathrm{~mm})$ : unmodified (A), modified with $0.5 \mathrm{ml}$ of $20 \mathrm{mM}$ sodium dodecylsulfate in water (B), modified with $0.5 \mathrm{ml}$ of $20 \mathrm{mM}$ sodium octadecylsulfate in methanol (C). Mobile phase, acetonitrile-water (50:50); flow rate, $8.0 \mu \mathrm{l} / \mathrm{min}$; wavelength of UV detection, $254 \mathrm{~nm}$; analytes, $10 \mathrm{ppm}$ uracil (1), 31 ppm naphthalene (2), $2.6 \mathrm{ppm}$ anthracene (3), $7.8 \mathrm{ppm}$ pyrene (4); injection volume, $0.15 \mu \mathrm{l}$.

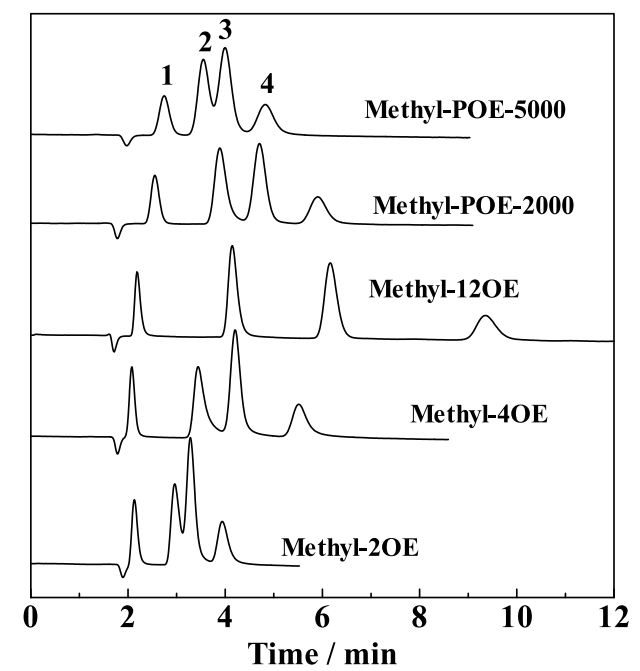

Fig. 8 Separation of inorganic anions on Methyl-POE-bonded stationary phases with different POE lengths. Columns, $100 \times 0.53 \mathrm{~mm}$ i.d.; mobile phase, $50 \mathrm{mM}$ sodium sulfate; flow rate, $8.0 \mu 1 / \mathrm{min}$; wavelength of UV detection, $210 \mathrm{~nm}$. Analytes: $0.1 \mathrm{mM}$ each of iodate (1), nitrate (2), iodide (3), thiocyanate (4); injection volume, $0.15 \mu$ l.

phase modified with sodium octadecylsulfate would be longer than that modified with sodium dodecylsulfate, the result was opposite. This is because sodium octadecylsulfate is not dissolved in water, and modification with sodium octadecylsulfate was achieved by using a strong solvent (methanol).

\section{Effect of chain length}

The effect of the POE chain length on the retention of anions was examined. Figure 8 demonstrates the separation of anions

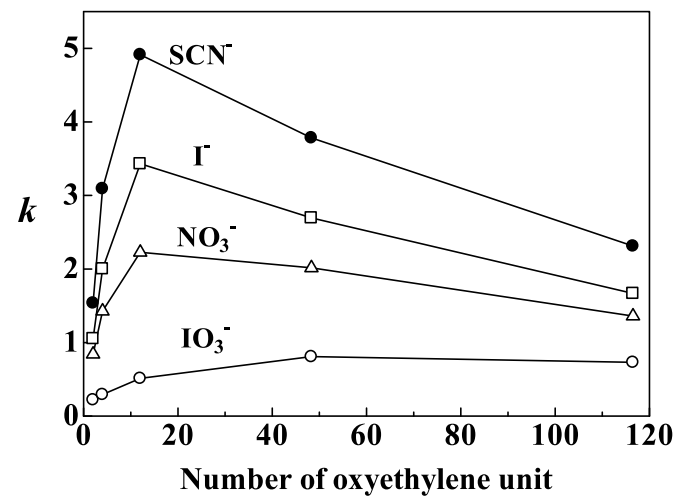

Fig. 9 The retention factor as a function of oxyethylene unit number. Operating conditions as in Fig. 8.

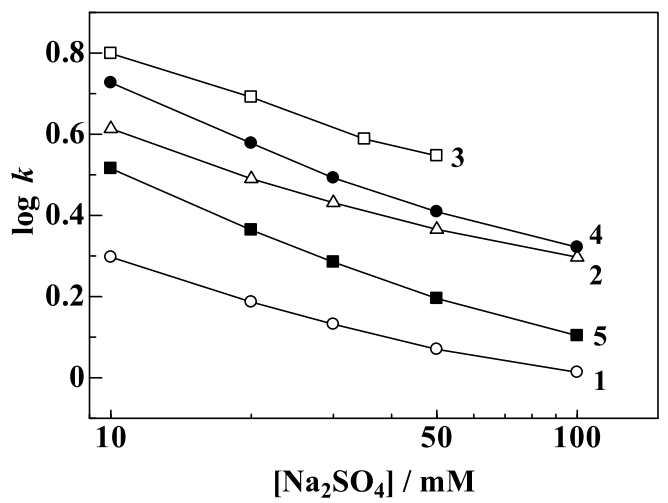

Fig. 10 Logarithm of the retention factor of thiocyanate versus the eluent concentration on the Methyl-POE stationary phases with different POE chain lengths. Columns: Methyl-2OE (1), Methyl-4OE (2), Methyl-12OE (3), Methyl-POE-2000 (4) and Methyl-POE-5000 (5); mobile phase, sodium sulfate; analyte, thiocyanate; other operating conditions as in Fig. 8.

on the POE stationary phases with the oxyethylene unit numbers of 2 -114. It can be seen that anions are retained on all of the stationary phases examined although the retention times were different. The retention factor of the anions is plotted as a function of the number of the oxyethylene unit in Fig. 9. It is found that the maximum retention is observed for the oxyethylene unit number of around 12. It can be expected that more eluent cations could be trapped among the POE chains with increasing chain length, leading to increase in the retention of anions for the oxyethylene unit number of $2-12$. However, it would be more difficult to adjust the location of the POE chain for much larger oxyethylene unit number, leading to the decrease in the amount of trapped eluent cations.

Figure 10 illustrates the logarithm of the retention factor of thiocyanate versus the sodium sulfate concentration as the eluent on the Methyl-POE stationary phases with different POE chain lengths, where almost linear relationships are observed between the above parameters. The slopes of the linear curves were $-0.28,-0.32,-0.37,-0.41$ and -0.41 for Methyl-2OE, 4OE, 12OE, POE-2000 and POE-5000, respectively. Theoretically, the slope value should be -0.5 under the condition in Fig. 10 as long as the anion exchange alone is involved in the retention. Actually, the observed slopes were shallower than the expected 


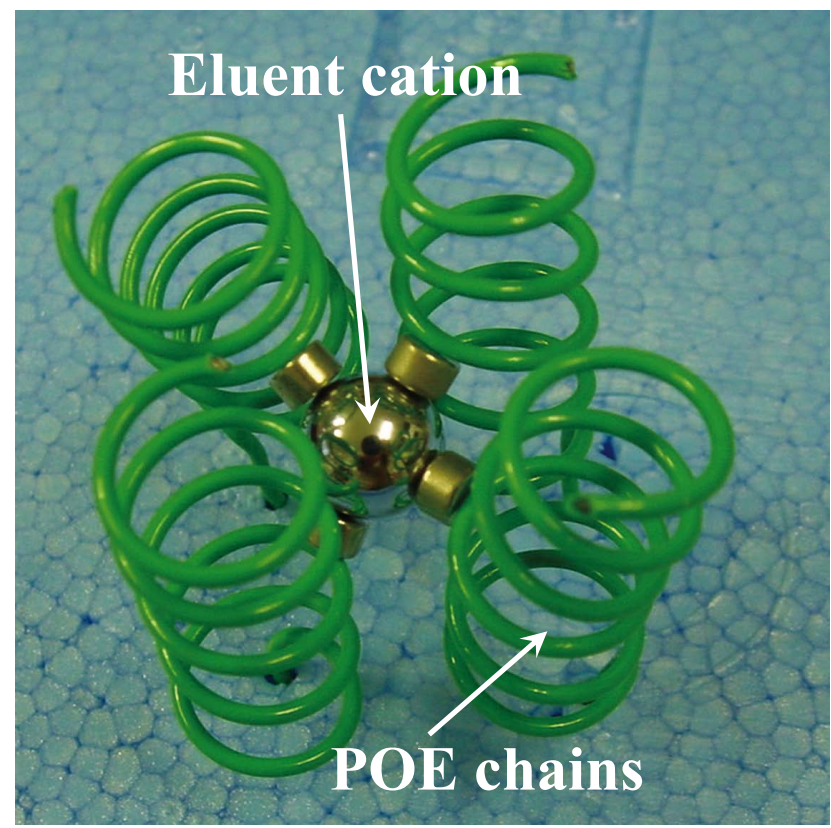

Fig. 11 Proposed cation-trapping structure.

ones. It was also found that the slope became deeper with increasing POE chain length. It should be noted that copper ions were trapped on these Methyl-POE stationary phases even for the Methyl-2OE stationary phase.

\section{Imaginary cation-trapping structure}

It is reported that POE chains could form a helix or coil conformation, depending on their molecular weight as well as on the solution and the temperature. ${ }^{9}$ It is therefore expected that the coil or helical POE chain could trap the eluent cation in a single coil or helical chain. However, considering that the Methyl-2OE stationary phase could also trap the eluent cation, where anion-exchange could take place, one finds that the eluent cation is trapped among multiple POE chains. The proposed cation-trapping structure is shown in Fig. 11. It is indicated that the eluent cation is trapped by ion-dipole interaction with the oxygen atoms of multiple POE chains, and the eluent and analyte anions can compete for the trapped cation. Inorganic anions can be therefore separated on the POE-bonded phases in the ion-exchange mode.

In addition, coated PEG stationary phases did not retain anions in the ion-exchange mode, ${ }^{3}$ but did retain them in the partition mode. This is because coated PEG molecules cannot take up the positions so that the eluent cations can be trapped among multiple PEG molecules.

\section{Conclusion}

The present POE-bonded stationary phases could work in the ion-exchange, RP and HILIC modes, depending on the eluent employed. The chemically-bonded POE chains could trap the eluent cations, this induced anion-exchange properties and enhanced hydrophobicity of the stationary phase.

\section{Acknowledgements}

This work was partially supported by Japan Science and Technology Agency, Research for Promoting Technological Seeds, No. 08-106, 2009.

\section{References}

1. H. Small and T. E. Miller, Jr., Anal. Chem., 1982, 54, 462.

2. L. Rong and T. Takeuchi, J. Chromatogr., A, 2004, 1042, 131.

3. L. Rong, L. W. Lim, and T. Takeuchi, Talanta, 2007, 72, 1625.

4. T. Takeuchi, B. Oktavia, and L. W. Lim, Anal. Bioanal. Chem., 2009, 393, 1267.

5. Z. Guo, Y. Liu, J. Xu, Q. Xu, X. Xue, F. Zhang, Y. Ke, X. Liang, and A. Lei, J. Chromatogr., A, 2008, 1191, 78.

6. F. Pellati, S. Benvenuti, and M. Melegari, J. Chromatogr., A, 2005, 1088, 205.

7. F. Cacciola, P. Jandera, Z. Hajdú, P. Česla, and L. Mondello, J. Chromatogr., A, 2007, 1149, 73.

8. M. L. Alessi, A. I. Norman, S. E. Knowlton, D. L. Ho, and S. C. Greer, Macromolecules, 2005, 38, 9333.

9. J. D. Lamb and R. G. Smith, J. Chromatogr., 1991, 546, 73.

10. T. Takeuchi and D. Ishii, J. Chromatogr., 1981, 213, 25. 